\title{
Spitzer's Identity and the Algebraic Birkhoff Decomposition in $\mathrm{PQFT}$
}

\author{
KURUSCH EBRAHIMI-FARD ${ }^{1}$ \\ Institut Henri Poincaré \\ 11, rue Pierre et Marie Curie \\ F-75231 Paris Cedex 05, France \\ and \\ Universität Bonn - Physikalisches Institut \\ Nussallee 12, D-53115 Bonn, Germany \\ LI GUO ${ }^{2}$ \\ Rutgers University \\ Department of Mathematics and Computer Science \\ Newark, NJ 07102, USA \\ DIRK KREIMER ${ }^{3}$ \\ C.N.R.S.-I.H.É.S. \\ Le Bois-Marie, 35, Route de Chartres \\ F-91440 Bures-sur-Yvette, France
}

July 11, 2004

\begin{abstract}
In this article we continue to explore the notion of Rota-Baxter algebras in the context of the Hopf algebraic approach to renormalization theory in perturbative quantum field theory. We show in very simple algebraic terms that the solutions of the recursively defined formulae for the Birkhoff factorization of regularized Hopf algebra characters, i.e. Feynman rules, naturally give a non-commutative generalization of the well-known Spitzer's identity. The underlying abstract algebraic structure is analyzed in terms of complete filtered Rota-Baxter algebras.
\end{abstract}

Keywords: Rota-Baxter algebras, Spitzer's identity, Baker-Campbell-Hausdorff formula, Bogoliubov's recursion, renormalization theory, Hopf algebra of graphs, Birkhoff decomposition.

\footnotetext{
${ }^{1}$ kurusch@ihes.fr

2liguo@newark.rutgers.edu

${ }^{3}$ kreimer@ihes.fr and dkreimer@bu.edu, Center for Math.Phys., Boston University.
} 


\section{Introduction}

The theory of Rota-Baxter type algebras has a long and interesting history. It was introduced by the American mathematician Glen Baxter in 1960 [4] in the context of fluctuations in probability theory. The subject was further explored especially by F. V. Atkinson [2], J. F. C. Kingman [19], P. Cartier [6] and others, but foremost by the mathematician Gian-Carlo Rota in his work in the late 1960s and early 1970s [27, 26] and later in his beautiful reviews [29, 30]. In the center of these works stood the category of commutative associative Rota-Baxter algebras and its free objects. Recently, one of us together with W. Keigher gave a very concise description of the latter in terms of a mixable shuffle product [16, 17, which provides a generalization of the classical shuffle product [13. As one of the main results of the above early work on (free) commutative Rota-Baxter algebras simple combinatorial and analytical proofs of Spitzer's identity were obtained. The latter is in its classical form a well-known object in probability theory having many applications.

Let us mention here that the Lie algebraic version of the Rota-Baxter relation plays under the name (modified) classical Yang-Baxter ${ }^{4}$ equation a prominent rôle in the theory of integrable systems [3, 28, 31, 32. Classical R-matrices, i.e. solutions of this equation, are connected to the Riemann-Hilbert problem and related factorization problems.

Recently the notion of Rota-Baxter algebra reappeared in the mathematics and, above all, physics literature. On the mathematics side we would like to underline its intimate link to Loday's dendriform algebra structures [1, 12, 14, 23, 24. From a physics viewpoint it appeared in the Hopf algebraic approach to the theory of renormalization in perturbative quantum field theory (pQFT). This approach provided a solid mathematical frame for renormalization theory in terms of combinatorial Hopf algebras of Feynman graphs [8, 9, 20, 21.

Here, we will dwell mainly on the latter aspect by showing that the recently given solutions to the recursively defined formulae for the algebraic Birkhoff factorization of regularized Hopf algebra characters in terms of a so-called BCH-recursion [10, 11] provide natural non-commutative generalizations of the above mentioned Spitzer's identity. We introduce the notion of complete filtered not necessarily commutative Rota-Baxter algebras to underline the abstract algebraic structure giving rise to this factorization. This approach allows us to derive in a fairly simple manner an alternative recursion for renormalized Feynman rules.

The paper is organized as follows. In Section 2 we collect some basic facts about (not necessarily commutative) Rota-Baxter algebras. Section 3 contains the non-commutative generalization of Spitzer's identity in the context of complete filtered Rota-Baxter algebras and an abstract algebraic formulation of Bogoliubov's recursion. Using the above results we finish this paper with a short review of the Birkhoff decomposition of regularized Hopf algebra characters. This turns out to be an example for the more general content of the forgoing section, placed in the context of the Hopf algebra approach to renormalization theory. It allows us to derive a new recursion formula for renormalized Feynman rules solely based on iterating the renormalized character $\phi_{+}$instead of the countertem $\phi_{-}$. We finish this article with a short summary and outlook.

\footnotetext{
${ }^{4}$ Here the relation is named after the physicists C.-N.Yang and Rodney Baxter.
} 


\section{Rota-Baxter algebras}

Let $\mathbb{K}$ be a field of characteristic 0 . By a $\mathbb{K}$-algebra we mean an associative algebra over $\mathbb{K}$ that is not necessarily unital nor commutative unless stated otherwise.

Definition 2.1. Let $\mathcal{A}$ be a $\mathbb{K}$-algebra with a $\mathbb{K}$-linear map $R: \mathcal{A} \rightarrow \mathcal{A}$. We call $\mathcal{A}$ a Rota-Baxter $\mathbb{K}$-algebra and $R$ a Rota-Baxter map (of weight $\theta \in \mathbb{K}$ ) if the operator $R$ holds the following Rota-Baxter relation of weight $\theta \in \mathbb{K}^{5}$ :

$$
R(x) R(y)+\theta R(x y)=R(R(x) y+x R(y)), \forall x, y \in \mathcal{A} .
$$

Remark 2.2. (0) Obviously, the above definition extends to non-associative algebras in general, and the field $\mathbb{K}$ may be replaced by an arbitrary commutative ring.

(1) In the rest of the paper we will fix the weight $\theta=1$, which is called the standard form or the Rota-Baxter relation.

(2) If $R$ fulfills the standard form of (11) then $\tilde{R}:=i d_{\mathcal{A}}-R$ fulfills the same Rota-Baxter relation.

(3) The ranges of $R$ and $\tilde{R}=i d_{\mathcal{A}}-R$ give subalgebras in $\mathcal{A}$.

(4) $R$ and $\tilde{R}=i d_{\mathcal{A}}-R$ fulfill the following mixed relations

$$
\begin{aligned}
R(x) \tilde{R}(y) & =R(x \tilde{R}(y))+\tilde{R}(R(x) y) \\
\tilde{R}(x) R(y) & =R(\tilde{R}(x) y)+\tilde{R}(x R(y)), \quad x, y \in \mathcal{A} .
\end{aligned}
$$

Example 2.3. (0) On the algebra of Laurent series $\left.\mathbb{C}\left[\epsilon^{-1}, \epsilon\right]\right]$ we have (up to automorphisms, ) only the following two Rota-Baxter maps $R^{(r)}, r=0,1$. Both are of weight $\theta=1$ and defined as follows. For $\left.\sum_{k=-m}^{\infty} c_{k} \epsilon^{k} \in \mathbb{C}\left[\epsilon^{-1}, \epsilon\right]\right]$ they give:

$$
R^{(r)}\left(\sum_{k=-m}^{\infty} c_{k} \epsilon^{k}\right):=\sum_{k=-m}^{-r} c_{k} \epsilon^{k}, r=0,1 .
$$

Within renormalization theory, so-called dimensional regularization together with the minimal subtraction scheme, i.e. $R_{M S}:=R^{(1)}$, play an important rôle [21].

(1) The case of a Rota-Baxter map of weight $\theta=0$, i.e. $R(x) R(y)=R(R(x) y+x R(y))$, naturally translates into the ordinary shuffle relation, and finds its most prominent example in the integration by parts rule for the Riemann integral. On the other hand Jackson's q-integral [29] gives a generalization of the Riemann integral to a Rota-Baxter map of weight $\theta=1-q$.

Proposition 2.4. In the case of the Rota-Baxter algebra $\mathcal{A}$ to be a Lie admissible $\mathbb{K}$ algebra, the Rota-Baxter relation naturally extends to the Lie algebra $\mathcal{L}_{\mathcal{A}}$ with commutator bracket $[x, y]:=x y-y x, \forall x, y \in \mathcal{A}$ :

$$
[R(x), R(y)]+R([x, y])=R([R(x), y]+[x, R(y)]) .
$$

\footnotetext{
${ }^{5}$ Some authors denote this relation in the form $R(x) R(y)=R(R(x) y+x R(y)+\lambda x y)$. So that $\lambda=-\theta$.
} 
Proposition 2.5. Let $\mathcal{A}$ be a Rota-Baxter algebra with Rota-Baxter map R. Equipped with the new product

$$
a *_{R} b:=R(a) b+a R(b)-a b,
$$

the vector space underlying $\mathcal{A}$ is again a Rota-Baxter algebra of the same type, denoted by $\mathcal{A}_{R}$.

The proof of this Proposition is a fairly easy exercise and follows directly from the identity (II) for $\theta=1$. We call this new Rota-Baxter algebra $\left(\mathcal{A}_{R}, R\right)$ the double of $\mathcal{A}$, and $*_{R}$ the double product.

Remark 2.6. (0) Let us remark here that this double construction appeared in a Lie algebraic context in 31], where the name was coined.

(1) The product $*_{R}$ can be written using $R$ and $\tilde{R}=i d_{\mathcal{A}}-R$ :

$$
a *_{R} b=R(a) b-a \tilde{R}(b),
$$

which can be interpreted in terms of the dendriform dialgebra structure of Loday [24].

(2) From the definition of the $*_{R}$ product in (6) it is obvious, that $R$ and $\tilde{R}=i d_{\mathcal{A}}-$ $R$ become an (not necessarily unital) algebra homomorphism and anti-homomorphism, respectively, from the double $\mathcal{A}_{R}$ to $\mathcal{A}$ :

$$
\begin{aligned}
& R\left(a *_{R} b\right)=R(a) R(b) \\
& \tilde{R}\left(a *_{R} b\right)=-\tilde{R}(a) \tilde{R}(b) .
\end{aligned}
$$

(3) As $\left(\mathcal{A}_{R}, *_{R}\right)$ is again of Rota-Baxter type, the above construction of the double extends to the so-called Rota-Baxter double hierarchy [11.

By definition, for the double product (6) we have

$$
a *_{R} b=R(a) R(b)-\tilde{R}(a) \tilde{R}(b)
$$

and so by (8),

$$
R(a) R(b)=R(R(a) R(b)-\tilde{R}(a) \tilde{R}(b)) .
$$

Inductively, this can be generalized to

$$
\prod_{i=1}^{n} R\left(x_{i}\right)=R\left(\prod_{i=1}^{n} R\left(x_{i}\right)-(-1)^{n} \prod_{i=1}^{n} \tilde{R}\left(x_{i}\right)\right), \quad x_{i} \in \mathcal{A}, i=1 \ldots n
$$

and then specialized to the following simple formula of Kingman which appeared in [19].

$$
R(u)^{n}=R\left(R(u)^{n}-(-\tilde{R}(u))^{n}\right), \quad u \in \mathcal{A} .
$$

\section{Non-commutative Spitzer's formula}

In the following, we do assume that an algebra in general is associative and unital, the unit will be denoted by 1 , but we do not assume that the algebra is commutative. 


\subsection{Spitzer's formula}

Spitzer's formula 33] is regarded as a remarkable stepping stone in the theory of sums of independent random variables in the fluctuation theory of probability. It was also the motivation for Baxter to define his identity [4]. The identity of Spitzer has the following algebraic formulation.

Theorem 3.1. 27] Let $(\mathcal{A}, R)$ be an unital commutative Rota-Baxter $\mathbb{Q}$-algebra of weight $\theta=1$. Then for $a \in \mathcal{A}$, we have

$$
\exp \left(R\left(\log (1-a x)^{-1}\right)\right)=\sum_{n=0}^{\infty} x^{n} \underbrace{R(R(R(\cdots(R(a) a) a) a))}_{n \text {-times }}
$$

in the ring of power series $\mathcal{A}[[x]]$.

For other than the combinatorial proofs of Spitzer and Baxter, we refer the interested reader to see [2, 6, 19, 27, 34].

Using our previous work [11] on the Birkhoff decomposition of regularized characters in the Hopf algebraic approach to renormalization theory in pQFT, we will derive a noncommutative version of Spitzer's formula. Quite remarkably, the proof presented here is similar to the one given in the commutative case by Kingman 19. Furthermore, once this formula is obtained, a simple but beautiful result of Atkinson in Theorem 3.6 applies to give us a new recursive formula back in the realm of Birkhoff decomposition in renormalization theory with respect to the so-called renormalized character, which we will describe in the next section.

We first consider Rota-Baxter algebras with a complete filtration. This setup allows us to apply our results to the Rota-Baxter algebra of renormalization introduced in [10]. The general case of Rota-Baxter algebras $\mathcal{A}$ will be treated by considering the power series ring $\mathcal{A}[[x]]$ in the commuting variable $x$.

\subsection{Complete Rota-Baxter algebras}

We first introduce the category of complete Rota-Baxter algebras.

Definition 3.2. $A$ filtered Rota-Baxter algebra is a Rota-Baxter algebra $(\mathcal{A}, R)$ together with a decreasing filtration $\mathcal{A}_{n}, n \geq 0$ of Rota-Baxter subalgebras. Thus we have

$$
\mathcal{A}_{n} \mathcal{A}_{m} \subseteq \mathcal{A}_{n+m}
$$

and

$$
R\left(\mathcal{A}_{n}\right) \subseteq \mathcal{A}_{n}
$$

Such a filtered Rota-Baxter algebra is called complete $^{6}$ if $\cap \mathcal{A}_{n}=0$ and if the resulting embedding

$$
\mathcal{A} \rightarrow \overline{\mathcal{A}}:=\lim _{\longleftarrow} \mathcal{A} / \mathcal{A}_{n}
$$

is an isomorphism.

\footnotetext{
${ }^{6}$ To avoid possible confusions, we alert the reader that in 17 the concept of complete filtered RotaBaxter algebras has been defined, where the filtration is canonically derived from the Rota-Baxter operator. That definition is not needed in this paper.
} 
By the completeness of the filtered Rota-Baxter algebra $(\mathcal{A}, R)$, the functions

$$
\begin{gathered}
\exp : \mathcal{A}_{1} \rightarrow 1+\mathcal{A}_{1}, \exp (a):=\sum_{n=0}^{\infty} \frac{a^{n}}{n !}, \\
\log : 1+\mathcal{A}_{1} \rightarrow \mathcal{A}_{1}, \log (1+a):=-\sum_{n=1}^{\infty} \frac{(-a)^{n}}{n}
\end{gathered}
$$

are well-defined. This has the following (classical) interpretation of Lie groups and Lie algebras.

$1+\mathcal{A}_{1}$ has a Lie group structure by the multiplication in $\mathcal{A}$, and $\mathcal{A}_{1}$ has a Lie algebra structure by the commutator bracket $[a, b]:=a b-b a$. Then the maps exp and log are the isomorphisms from the Lie algebra to the Lie group and its inverse.

Example 3.3. For the Hopf algebra $\mathcal{H}_{F G}$ of Feynman graphs (or rooted trees) and the ring of Laurent series $\left.\mathcal{A}:=\mathbb{C}\left[\epsilon^{-1}, \epsilon\right]\right]$ with the Rota-Baxter operator defined to be the projection to the pole part, i.e. $\left.R:=R^{(1)}: \mathbb{C}\left[\epsilon^{-1}, \epsilon\right]\right] \rightarrow \epsilon^{-1} \mathbb{C}\left[\epsilon^{-1}\right]$ in (44), the algebra $L\left(\mathcal{H}_{F G}, \mathcal{A}\right)$ with the convolution product and lifted Rota-Baxter map $\mathcal{R}: L\left(\mathcal{H}_{F G}, \mathcal{A}\right) \rightarrow$ $L\left(\mathcal{H}_{F G}, \mathcal{A}\right)$ is a complete Rota-Baxter algebra [8, 15, 20]. See [25, II.3.3.] for the proof. Further in this setting $1+A_{1}$ is the group of (regularized) characters and $A_{1}$ is the Lie algebra of infinitesimal characters.

For $a \in \mathcal{A}$, inductively define

$$
(R a)^{[n+1]}:=R\left((R a)^{[n]} a\right) \text { and }(R a)^{\{n+1\}}:=R\left(a(R a)^{\{n\}}\right)
$$

with the convention that $(R a)^{[1]}=R(a)=(R a)^{\{1\}}$ and $(R a)^{[0]}=1=(R a)^{\{0\}}$.

Also by the completeness, there is a unique map $\chi: \mathcal{A}_{1} \rightarrow \mathcal{A}_{1}$ that satisfies the equation

$$
\chi(a)=a-B C H(R(\chi(a)), \tilde{R}(\chi(a)))
$$

which was introduced in [11] and will be coined as $B C H$-recursion for short. Here $B C H(x, y)$ denotes the Baker-Campbell-Hausdorff formula such that

$$
\exp (x) \exp (y)=\exp (x+y+B C H(x, y))
$$

which is a power series in $x, y$ of degree 2. Relation (13) was used in our approach to the algebraic Birkhoff factorization, in connection with a classical R-matrix notion coming from a Lie Rota-Baxter relation (5), see Section 4 .

We call it the $B C H$-recursion since $\chi(a)$ is defined to be $\lim _{n \rightarrow \infty} \chi_{n}(a)$ where

$$
\begin{aligned}
\chi_{0}(a) & =a \\
\chi_{n+1}(a) & =a-B C H\left(R\left(\chi_{n}(a)\right), \tilde{R}\left(\chi_{n}(a)\right)\right) .
\end{aligned}
$$

To see why this gives the unique solution to recursion relation (13), we first define for $a \in \mathcal{A}, \Lambda: \mathcal{A} \rightarrow \mathcal{A}[11$

$$
\Lambda(a):=B C H(R(a), \tilde{R}(a))
$$


Then for $s \in A_{n}, n \geq 1, \Lambda(a+s)$ is $\Lambda(a)$ plus a sum in which each term has $s$ occurring at least once, and hence is contained in $A_{n+1}$. Thus we have

$$
\Lambda\left(a \bmod A_{n}\right) \equiv \Lambda(a) \bmod A_{n+1}
$$

Now we have

$$
\chi_{1}(a)=a+\Lambda\left(\chi_{0}(a)\right)=a+\Lambda(a) \equiv a \equiv \chi_{0}(a) \bmod A_{2} .
$$

By induction on $n$ and (14), we have

$$
\begin{aligned}
\chi_{n+1}(a) & =a+\Lambda\left(\chi_{n}(a)\right) \\
& \equiv a+\Lambda\left(\chi_{n-1}(a) \bmod A_{n+1}\right) \\
& \equiv a+\Lambda\left(\chi_{n-1}(a)\right) \bmod A_{n+2} \\
& \equiv \chi_{n}(a) \bmod A_{n+2} .
\end{aligned}
$$

Thus $\lim _{n \rightarrow \infty} \chi_{n}(a)$ exists and is a solution of (13).

Suppose $b$ is another solution. Then, as above, we have

$$
\chi_{0}(a)=a \equiv a+\Lambda(b) \equiv b \quad \bmod A_{2} .
$$

Induction on $n$ gives the following

$$
\begin{aligned}
\chi_{n+1}(a) & =a+\Lambda\left(\chi_{n}(a)\right) \\
& \equiv a+\Lambda\left(b \bmod A_{n+2}\right) \\
& \equiv a+\Lambda(b) \bmod A_{n+3} \\
& \equiv b \bmod A_{n+3} .
\end{aligned}
$$

Thus $b=\lim _{n \rightarrow \infty} \chi_{n}(a)$. The reader may find it helpful to consult the nice expository work of Manchon [25] for a more conceptual proof in the context of Lie algebras.

Theorem 3.4. Let $\left(\mathcal{A}, R, \mathcal{A}_{n}\right)$ be a complete filtered Rota-Baxter algebra of weight $\theta=1$. Let $a \in \mathcal{A}_{1}$.

1. The equation

$$
b=1-R(b a)
$$

has a unique solution

$$
b=\exp (-R(\chi(\log (1+a))))
$$

2. The equation

$$
b=1-\tilde{R}(a b)
$$

has a unique solution

$$
b=\exp (-\tilde{R}(\chi(\log (1+a))))
$$


Proof. We only need to verify for the first equation. The proof for the second equation is similar.

Since $a$ is in $A_{1}$ and $R$ preserves the filtration, the series

$$
b=1+R(a)+R(R(a) a)+\cdots+(R a)^{[n]}+\cdots
$$

defines a unique element in $\mathcal{A}$ and is easily seen to be a solution of (15). Conversely, if $c \in \mathcal{A}$ is a solution of (15), then by iterated substitution, we have

$$
c=1+R(a)+R(R(a) a)+\cdots+(R a)^{[n]}+\cdots .
$$

Therefore, the equation (15) has a unique solution.

To verify that (16) gives the solution, take $u:=\log (1+a), a \in \mathcal{A}_{1}$. Using (11), for our chosen $b$ we have

$$
\begin{aligned}
& \exp (-R(\chi(\log (1+a))))=\exp (-R(\chi(u))) \\
& =\sum_{n=0}^{\infty} \frac{(-R(\chi(u)))^{n}}{n !} \\
& =1+R\left(\sum_{n=1}^{\infty} \frac{(-1)^{n}}{n !}\left((R(\chi(u)))^{n}-(-\tilde{R}(\chi(u)))^{n}\right)\right) \\
& =1+R\left(\sum_{n=0}^{\infty} \frac{(-1)^{n}}{n !}(R(\chi(u)))^{n}-\sum_{n=0}^{\infty} \frac{(-1)^{n}}{n !}(-\tilde{R}(\chi(u)))^{n}\right) \\
& =1+R(\exp (-R(\chi(u)))-\exp (\tilde{R}(\chi(u)))) .
\end{aligned}
$$

By the definition of the $B C H$-recursion $\chi$ in equation (13), we have

$$
\begin{aligned}
& \exp (R(\chi(u))) \exp (\tilde{R}(\chi(u))) \\
= & \exp (R(\chi(u))+\tilde{R}(\chi(u))+B C H(R(\chi(u)), \tilde{R}(\chi(u)))) \\
= & \exp (\chi(u)+B C H(R(\chi(u)), \tilde{R}(\chi(u)))) \\
= & \exp (u) .
\end{aligned}
$$

Thus

$$
\begin{aligned}
& \exp (-R(\chi(\log (1+a)))) \\
= & 1+R(\exp (-R(\chi(u)))-\exp (-R(\chi(u))) \exp (u)) \\
= & 1+R(\exp (-R(\chi(u)))(1-\exp (u))) \\
= & 1+R(\exp (-R(\chi(\log (1+a))))(1-\exp (\log (1+a)))) \\
= & 1-R(\exp (-R(\chi(\log (1+a))) a)
\end{aligned}
$$

This verifies the first equation. 
Corollary 3.5. Let $\left(\mathcal{A}, R, \mathcal{A}_{n}\right)$ be a complete filtered Rota-Baxter algebra of weight $\theta=1$. For $a \in \mathcal{A}_{1}$, we have

$$
\begin{aligned}
& \sum_{n=0}^{\infty}(R a)^{[n]}=\exp (-R(\chi(\log (1+a)))) \\
& \sum_{n=0}^{\infty}(\tilde{R} a)^{\{n\}}=\exp (-\tilde{R}(\chi(\log (1+a))))
\end{aligned}
$$

Proof. By Theorem 3.4 and its proof, both sides of (19) are solutions of (15). This proves (19).

The proof of (20) is the same, by considering solutions of the recursive equation (17)

For later reference, we record here a simple and attractive theorem of Atkinson 2 whose proof just uses relations (2) and (3).

Theorem 3.6. Let $(\mathcal{A}, R)$ be an associative unital but not necessarily commutative RotaBaxter algebra. Assume $b$ and $b^{\prime}$ to be solutions of the recursive equations (15) and (17), then

$$
b(1+a) b^{\prime}=1
$$

We now prove the Birkhoff decomposition of filtered Rota-Baxter algebras.

Theorem 3.7. Let $(\mathcal{A}, R)$ be an associative unital complete Rota-Baxter algebra with filtration $A_{n}, n \geq 0$. The following conditions are equivalent.

(i) $R$ is idempotent: $R^{2}=R$ when restricted to $A_{1}$.

(ii) There is a direct product decomposition of algebras

$$
A_{1}=R\left(A_{1}\right) \times \tilde{R}\left(A_{1}\right) .
$$

(iii) There is a direct product decomposition of groups

$$
\left(1+A_{1}\right)=\left(1+R\left(A_{1}\right)\right) \times\left(1+\tilde{R}\left(A_{1}\right)\right) .
$$

Remark 3.8. Under the assumption in $(i)$, the statement in $(i i)$ is the Atkinson decomposition [2] and the statement in (iii) specializes to give the uniqueness of the Birkhoff decomposition of Connes and Kreimer. See Section 4 for details. 
Proof. (1) $\Leftrightarrow(2)$ is clear and does not need the completeness assumption.

$(2) \Rightarrow(3)$ : We just need to show that, for each $a \in A_{1}$, there is a unique $c \in R\left(A_{1}\right)$ and a unique $\tilde{c} \in \tilde{R}\left(A_{1}\right)$ such that

$$
1+a=(1+c)(1+\tilde{c}) .
$$

Let $a \in A_{1}$ be given, and let $b$ and $\tilde{b}$ be the solution of (15) and (17) respectively. Then by Theorem 3.6, we have

$$
b(1+a) \tilde{b}=1
$$

By their constructions and (89), we have $b=1-b_{1}$ and $\tilde{b}=1-\tilde{b}_{1}$ for $b_{1} \in R\left(A_{1}\right)$ and $\tilde{b}_{1} \in \tilde{R}\left(A_{1}\right)$. Thus

$$
\begin{aligned}
& b^{-1}=1+b_{1}+b_{1}^{2}+\cdots \in 1+R\left(A_{1}\right), \\
& \tilde{b}^{-1}=1+\tilde{b}_{1}+\tilde{b}_{1}^{2}+\cdots \in 1+\tilde{R}\left(A_{1}\right) .
\end{aligned}
$$

This proves the existence.

For the uniqueness, suppose we have

$$
1+a=(1+c)(1+\tilde{c})=(1+d)(1+\tilde{d})
$$

with $c, d \in R\left(A_{1}\right)$ and $\tilde{c}, \tilde{d} \in \tilde{R}\left(A_{1}\right)$. Then

$$
(1+d)^{-1}(1+c)=(1+\tilde{d})(1+\tilde{c})^{-1}
$$

which is in $\left(1+R\left(A_{1}\right)\right) \cap\left(1+\tilde{R}\left(A_{1}\right)\right)$. But this intersection is $\{1\}$ because

$$
1+R(d)=1+\tilde{R}\left(d^{\prime}\right) \Rightarrow R(d)=\tilde{R}\left(d^{\prime}\right) \Rightarrow R(d)=0 .
$$

$(3) \Rightarrow(2)$ : Since $R+\tilde{R}=\mathrm{id}$, we have $A_{1}=R\left(A_{1}\right)+\tilde{R}\left(A_{1}\right)$. So we just need to show $R\left(A_{1}\right) \cap \tilde{R}\left(A_{1}\right)=0$. This is true if and only if $\left(1+R\left(A_{1}\right)\right) \cap\left(1+\tilde{R}\left(A_{1}\right)\right)=\{1\}$..

\subsection{Algebraic Bogoliubov map}

For $a \in A_{1}$, let $a_{-}$be the unique solution of $b=1-R(b a)$ from Theorem 3.4 and let

$$
\gamma(a)=a_{-} a
$$

Similarly, let $\tilde{a}$ be the unique solution of $b=1-\tilde{R}(a b)$ and let

$$
\tilde{\gamma}(a)=a \tilde{a}
$$

By Proposition 2.5. $A_{1}$ with the product $*_{R}$ is still a complete algebra. Define

$$
\exp _{R}: A_{1} \rightarrow 1+A_{1}, \exp _{R}(a):=\sum_{n=0}^{\infty} \frac{a^{*} R^{n}}{n !}
$$

where $a^{*_{R} n}$ is the $n$-th power of $a$ under the product $*_{R}$. 
Theorem 3.9. The following diagram commutes.

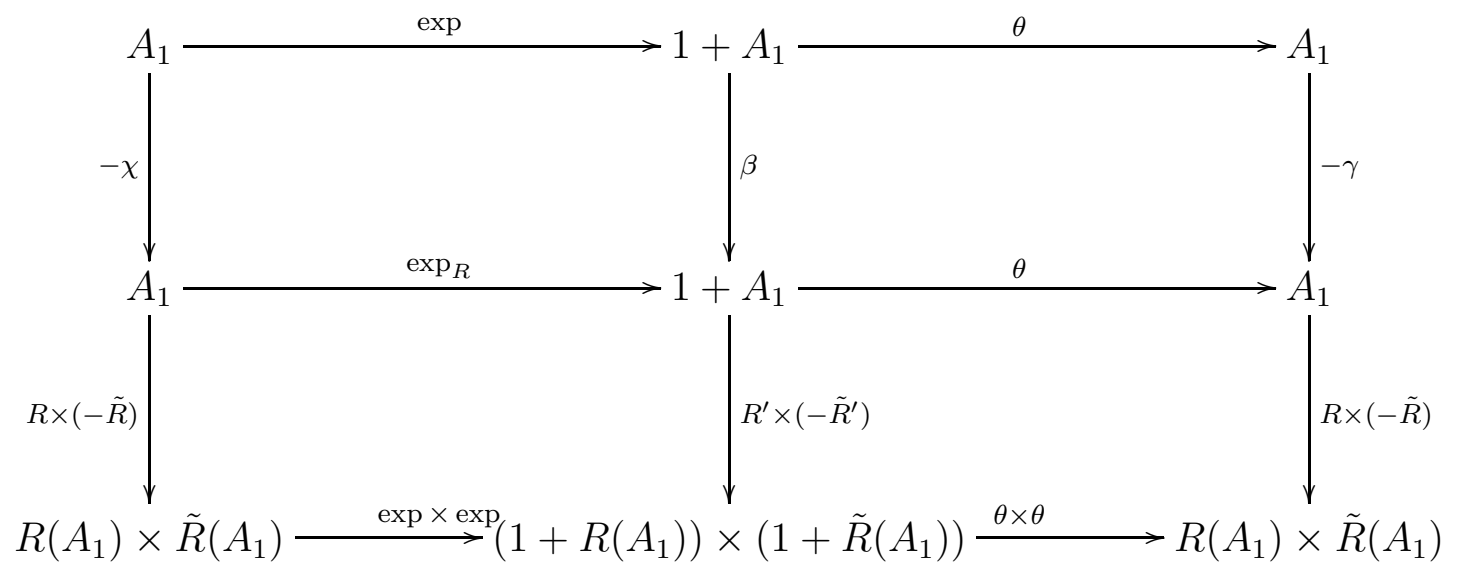

Here $\theta(x)=x-1$ and $\beta$ is defined to be the composite

$$
\beta=\theta^{-1} \circ(-\gamma) \circ \theta
$$

So $\beta(c)=1-\gamma(c-1)$. Similarly define $R^{\prime} \times\left(-\tilde{R}^{\prime}\right)$. So

$$
\begin{array}{ll}
R^{\prime}: & 1+A_{1} \rightarrow 1+A_{1}, a \mapsto 1+R(a-1), \\
\tilde{R}^{\prime}: & 1+A_{1} \rightarrow 1+A_{1}, \quad a \mapsto 1-\tilde{R}(a-1) .
\end{array}
$$

We call the map $\beta$ the algebraic Bogoliubov because it gives the Bogoliubov map in renormalization theory.

Proof. We only need to prove the commutativity of the upper half and the lower half of the diagram. By the way the two maps in the middle column are defined and by the bijectivity of the horizontal maps in the right half of the diagram, it follows that the other squares are also commutative.

Verifying the commutativity of the top half means to verify

$$
-\gamma \circ \theta \circ \exp (u)=\theta \circ \exp _{R} \circ(-\chi(u)) \text {, }
$$

that is,

$$
-\gamma(\exp (u)-1)=\exp _{R}(-\chi(u))-1 .
$$

By Theorem 3.4, and reversing the derivations in its proof, we have

$$
\begin{aligned}
-\gamma(\exp (u)-1) & =-\exp (-R(\chi(u))(\exp (u)-1) \\
& =-\exp (-R(\chi(u))) \exp (u)-\exp (-R(\chi(u))) \\
& =\exp (-R(\chi(u)))-\exp (\tilde{R}(\chi(u))) .
\end{aligned}
$$

By (8) and (9) we obtain

$$
R\left(\exp _{R}(u)\right)=\exp (R(u))+R(1)-1
$$




$$
\tilde{R}\left(\exp _{R}(u)\right)=-\exp (-\tilde{R}(u))+\tilde{R}(1)+1 \text {. }
$$

Thus the last term of the earlier equation is

$$
\begin{aligned}
& R\left(\exp _{R}(-\chi(u))\right)+1-R(1)+\tilde{R}\left(\exp _{R}(-\chi(u))\right)-1-\tilde{R}(1) \\
= & \exp _{R}(-\chi(u))-1
\end{aligned}
$$

as is desired.

Verifying the commutativity of the lower half of the diagram means to verify the two equations

$$
\exp (R(u))-1=R\left(\exp _{R}(u)-1\right), \quad \exp (-\tilde{R}(u))-1=-\tilde{R}\left(\exp _{R}(u)-1\right)
$$

which are immediately from (22) and (23).

\subsection{General Rota-Baxter algebras}

Now let $(\mathcal{A}, R)$ be any Rota-Baxter algebra of weight $\theta=1$. Consider the power series $\operatorname{ring} \mathcal{A}[[x]]$ on one (commuting) variable x. So $\mathcal{A}[[x]]=\mathbb{Z}[[x]] \otimes \mathcal{A}$. Define an operator,

$$
\mathcal{R}: \mathcal{A}[[x]] \rightarrow \mathcal{A}[[x]], \mathcal{R}\left(\sum_{n=0}^{\infty} a_{n} x^{n}\right)=\sum_{n=0}^{\infty} R\left(a_{n}\right) x^{n}
$$

Lemma 3.10. $(\mathcal{A}[[x]], \mathcal{R})$ is a Rota-Baxter algebra.

Proof. This is a straight forward verification. For $f=\sum_{n} a_{n} x^{n}, g=\sum_{m} b_{m} x^{m}$, we have

$$
\begin{aligned}
\mathcal{R}(f) \mathcal{R}(g)= & \left(\sum_{n} R\left(a_{n}\right) x^{n}\right)\left(\sum_{m} R\left(b_{m}\right) x^{m}\right) \\
= & \sum_{n, m} R\left(a_{n}\right) R\left(b_{m}\right) x^{m+n} \\
= & \sum_{n, m}\left(R\left(R\left(a_{n}\right) b_{m}\right)+R\left(a_{n} R\left(b_{m}\right)\right)-R\left(a_{n} b_{m}\right)\right) x^{m+n} \\
= & \mathcal{R}\left(\left(\sum_{n} R\left(a_{n}\right) x^{n}\right)\left(\sum_{m} b_{m} x^{m}\right)\right)+\mathcal{R}\left(\left(\sum_{n} a_{n} x^{n}\right)\left(\sum_{m} R\left(b_{m}\right) x^{m}\right)\right) \\
& -\mathcal{R}\left(\left(\sum_{n} a_{n} x^{n}\right)\left(\sum_{m} b_{m} x^{m}\right)\right) \\
= & \mathcal{R}(\mathcal{R}(f) g)+\mathcal{R}(f \mathcal{R}(g))-\mathcal{R}(f g) .
\end{aligned}
$$

Now it is easy to verify that, with the filtration

$$
\mathcal{A}_{n}:=x^{n} \mathcal{A}[[x]], n \geq 0,
$$

$\mathcal{A}[[x]]$ is a complete Rota-Baxter algebra. By Theorem 3.4, we have 
Corollary 3.11. For $a \in \mathcal{A}$, we therefore have

$$
\sum_{n=0}^{\infty}(\mathcal{R}(a x))^{[n]}=\exp (-\mathcal{R}(\chi(\log (1+a x))))
$$

Remark 3.12. Obviously, for $\mathcal{A}$ being commutative, we have $\chi(a)=a$, and relation (19) just reduces to the classical Spitzer's identity. Our result therefore is the natural non-commutative generalization of this well-known identity.

By comparing coefficients of similar powers of $x$ on the two sides of the equation (24), we obtain identities in Rota-Baxter algebras that are not necessarily commutative.

\section{Birkhoff decomposition in renormalization theory}

Now we consider the case when the complete Rota-Baxter algebra is as in Example 3.3 We will use the notations in articles [10] and [11]. For a general review on the Hopf algebraic approach to renormalization theory in pQFT, we refer the reader to the original work [7, 8, 20, 21]. For a recent and elaborate review of the Connes-Kreimer work on renormalization theory, we refer the reader to the work by Manchon [25].

Kreimer and later Connes and Kreimer were able to uncover the mathematical content underlying the algebraic combinatorial process of renormalization theory in pQFT, by organizing the combinatorics in terms of a combinatorial, i.e. graded connected Hopf algebra structure on Feynman graphs, denoted by $\mathcal{H}_{F G}$. Furthermore, by interpreting Feynman rules as regularized characters, i.e. multiplicative maps from the above Hopf algebra of Feynman graphs into an associative unital and commutative Rota-Baxter algebra, the process of renormalization became a Birkhoff decomposition of these characters.

We will denote the space of linear functionals from $\mathcal{H}_{F G}$ into the Rota-Baxter algebra $(\mathcal{A}, R)$ by $L\left(\mathcal{H}_{F G}, \mathcal{A}\right) . \quad L\left(\mathcal{H}_{F G}, \mathcal{A}\right)$ carries the structure of an associative unital noncommutative algebra with respect the convolution product, denoted by

$$
f \star g:=m_{\mathcal{A}}(f \otimes g) \Delta, f, g \in L\left(\mathcal{H}_{F G}, \mathcal{A}\right) .
$$

Here $\Delta$ denotes the coproduct in $\mathcal{H}_{F G}$. The unit in $L\left(\mathcal{H}_{F G}, \mathcal{A}\right)$ is given by the counit $\epsilon: \mathcal{H}_{F G} \rightarrow 1 \mathbb{K}$. Let $\phi$ be a regularized character, i.e. an element in the group $G \subset$ $L\left(\mathcal{H}_{F G}, \mathcal{A}\right)$, generated by the infinitesimal characters forming a Lie algebra $g \subset L\left(\mathcal{H}_{F G}, \mathcal{A}\right)$. We then lift the Rota-Baxter map $R: \mathcal{A} \rightarrow \mathcal{A}$ to the algebra $L\left(\mathcal{H}_{F G}, \mathcal{A}\right)$, see Proposition (4.1) below.

In [8], it was shown that for arbitrary $\phi \in G$ there exist two unique characters, defined recursively for $\Gamma \in \operatorname{ker}(\epsilon) \subset \mathcal{H}_{F G}$ by

$$
\phi_{ \pm}: \mathcal{H}_{F G} \rightarrow \mathcal{A},\left\{\begin{array}{l}
\phi_{-}(\Gamma):=-R\left[\phi(\Gamma)+\sum_{(\Gamma)}^{\prime} \phi_{-}\left(\Gamma^{\prime}\right) \phi\left(\Gamma^{\prime \prime}\right)\right], \\
\phi_{+}(\Gamma):=\tilde{R}\left[\phi(\Gamma)+\sum_{(\Gamma)}^{\prime} \phi_{-}\left(\Gamma^{\prime}\right) \phi\left(\Gamma^{\prime \prime}\right)\right], \quad \text { and } \\
\phi_{ \pm}(1):=1
\end{array}\right.
$$

such that

$$
\phi=\phi_{-}^{-1} \star \phi_{+}
$$


Here we used Sweedler's notation, $\Delta(\Gamma):=\Gamma \otimes 1+1 \otimes \Gamma+\sum_{(\Gamma)}^{\prime} \Gamma^{\prime} \otimes \Gamma^{\prime \prime}$ for $\Gamma \in \mathcal{H}_{F G}$. The character $S_{R}^{\phi}:=\phi_{-}$was called twisted antipode, and provides the counterterm. The so-called renormalized character $\phi_{+}$gives the renormalized Feynman rules. To proof the multiplicativity of $\phi_{-}$and $\phi_{+}$essential use of the Rota-Baxter structure on the target space $\mathcal{A}$ of the characters was made. Using the following

Proposition 4.1. [10] Define the linear map $\mathcal{R}: L\left(\mathcal{H}_{F G}, \mathcal{A}\right) \rightarrow L\left(\mathcal{H}_{F G}, \mathcal{A}\right)$ by $f \mapsto$ $\mathcal{R}(f):=R \circ f: \mathcal{H}_{F G} \rightarrow R(\mathcal{A})$. Then $L\left(\mathcal{H}_{F G}, \mathcal{A}\right)$ becomes an associative, unital noncommutative Rota-Baxter algebra. The Lie algebra of infinitesimal characters $g \subset L\left(\mathcal{H}_{F G}, \mathcal{A}\right)$ becomes a Lie Rota-Baxter algebra, i.e. for $Z^{\prime}, Z^{\prime \prime} \in g$,

$$
\left[\mathcal{R}\left(Z^{\prime}\right), \mathcal{R}\left(Z^{\prime \prime}\right)\right]=\mathcal{R}\left(\left[Z^{\prime}, \mathcal{R}\left(Z^{\prime \prime}\right)\right]\right)+\mathcal{R}\left(\left[\mathcal{R}\left(Z^{\prime}\right), Z^{\prime \prime}\right]\right)-\mathcal{R}\left(\left[Z^{\prime}, Z^{\prime \prime}\right]\right) .
$$

We can write equivalently, $\phi_{-}$in terms of the recursive equation

$$
\phi_{-}=\epsilon-\mathcal{R}\left[\phi_{-} \star(\phi \circ J)\right],
$$

where $J$, the projector onto the augmentation ideal $\operatorname{ker}(\epsilon)$, is defined in terms of the unit map $\eta: 1 \mathbb{K} \rightarrow \mathcal{H}_{F G}, J:=i d_{\mathcal{H}_{F G}}-\eta \epsilon$. Note that by linearity of $\phi$ we have

$$
(\epsilon+\phi \circ J)=\phi .
$$

Let $\phi \in G$ be generated by $Z \in g$, i.e. $\phi=\exp ^{\star}(Z)$. So by Theorem 3.4 the recursion (28) for $\phi_{-}$is solved by

$$
\begin{aligned}
\phi_{-} & =\exp ^{\star}\left(-\mathcal{R}\left(\chi\left(\log ^{\star}(\epsilon+\phi \circ J)\right)\right)\right) \\
& =\exp ^{\star}(-\mathcal{R}(\chi(Z)))
\end{aligned}
$$

as proved in [1].

We now let $\tilde{\phi}$ be defined by the recursive equation

$$
\tilde{\phi}=\epsilon-\tilde{\mathcal{R}}[(\phi \circ J) \star \tilde{\phi}] .
$$

So by Theorem 3.6. we have

$$
\phi_{-} \star \phi \star \tilde{\phi}=\epsilon .
$$

On the other hand, following (26), it is well-known that, for the unique renormalized character $\phi_{+}$, we have

$$
\phi_{-} \star \phi \star \phi_{+}^{-1}=\epsilon .
$$

Since both equations hold in the Lie group $G$ of regularized characters, we must have

$$
\begin{aligned}
\tilde{\phi} & =\phi_{+}^{-1} \\
& =\exp ^{\star}(-\tilde{\mathcal{R}}(\chi(Z))) .
\end{aligned}
$$

The second equality follows by Theorem 3.4 equation (18) and was shown for $\phi_{+}$directly in [11. This simple result implies a new recursive relation for $\phi_{+}$in terms of $\tilde{\mathcal{R}}$

$$
\phi_{+}=\epsilon-\tilde{\mathcal{R}}\left[\phi_{+} \star\left(\phi^{-1} \circ J\right)\right] .
$$


Note that this result is completely natural. The antipode $S\left(S^{2}=\mathrm{id}\right)$ can be written in terms of the projector $J$ as

$$
S=-m \circ(S \otimes J) \circ \Delta=-m \circ(J \otimes S) \circ \Delta .
$$

Iterating $\phi_{-}$on the left hand side of the tensor product, it was used to deform the character $\phi \circ S$ to the counterterm character $\phi_{-}$. But one naturally expects that one also can derive the forest formula by recursing $\phi_{+}$, and this is what the above formula achieves. The appearance of $\phi^{-1}$ then instead of $\phi$ compensates for the minus sign in front of $\tilde{\mathcal{R}}$, making use of the very exponentiation in (33).

Also, we remind ourselves that the renormalized character is a character in the image of $\tilde{\mathcal{R}}$, where, $\tilde{\mathcal{R}}$ acts on the Bogoliubov character, a map which replaces all subdivergences by their evaluation under $\phi_{+}$, a fact guaranteed by the structure of the Hochschild cohomology of such Hopf algebras [5, 22. Thus, one naturally recurses $\phi_{+}$in terms of itself, a fact evident also in the basic structure of renormalized Dyson-Schwinger equations, which can be completely written in terms of themselves. The above formula makes that fact self-evident on a combinatorial level.

Summarizing, by the above argument we find naturally the following two recursions for the factors of the Birkhoff decomposition of a character $\phi$ :

$$
\phi_{+}=\epsilon-\tilde{\mathcal{R}}\left[\phi_{+} \star\left(\phi^{-1} \circ J\right)\right] \text { and } \phi_{-}=\epsilon-\mathcal{R}\left[\phi_{-} \star(\phi \circ J)\right] .
$$

Using the augmentation ideal projector $J:=i d_{\mathcal{H}_{F G}}-\eta \epsilon$ we can derive the simple identity

$$
\begin{aligned}
\phi \star\left(\phi^{-1} \circ J\right) & =\phi \star \phi \circ S \circ J \\
& =\phi \star \phi \circ S \circ\left(i d_{\mathcal{H}_{F G}}-\eta \epsilon\right) \\
& =\phi \star \phi \circ S-\phi \star(\phi \circ S \circ \eta \epsilon) \\
& =\epsilon-\phi=-\phi \circ J,
\end{aligned}
$$

which allows us to show, using $\phi=\phi_{-}^{-1} \star \phi_{+}$, that

$$
\begin{aligned}
-\phi_{+} \star\left(\phi^{-1} \circ J\right) & =\phi_{+} \star \phi^{-1} \star(\phi \circ J) \\
& =\phi_{+} \star \phi_{+}^{-1} \star \phi_{-} \star(\phi \circ J) \\
& =\phi_{-} \star \phi \circ J .
\end{aligned}
$$

This allows us to get back the original $\phi_{+}$-recursion (25) in terms of the Bogoliubov character [11], i.e. Bogoliubov's R-map, defined via the double product $\star_{\mathcal{R}}, \exp ^{\star_{\mathcal{R}}}(\chi(Z))=$ $\phi_{-} \star \phi \circ J$

$$
\phi_{+}=\epsilon+\tilde{\mathcal{R}}\left[\phi_{-} \star(\phi \circ J)\right] .
$$

Let us for the sake of clarity compare the above results in the setting of combinatorial Hopf algebras and regularized characters with the findings of Section 3, i.e. general filtered Rota-Baxter algebras. To clearly show the connection, we display the following "dictionary". We fix a character $\phi: \mathcal{H}_{F G} \rightarrow \mathcal{A}$ in $G$ and let $a:=\phi \circ J$. In the following table, entries in the left column are results proved earlier in this paper for general complete 
filtered Rota-Baxter algebras, and entries in the right column are their interpretations in the non-commutative associative unital Rota-Baxter algebra $\left(L\left(\mathcal{H}_{F G}, \mathcal{A}\right), \mathcal{R}\right)$.

$$
\begin{array}{ll}
a & \phi \circ J \\
a_{-}=1-R\left(a_{-} a\right) & \phi_{-}=\epsilon-\mathcal{R}\left(\phi_{-} \star(\phi \circ J)\right) \\
\tilde{a}=1-\tilde{R}(a \tilde{a}) & \tilde{\phi}=\epsilon-\tilde{\mathcal{R}}((\phi \circ J) \star \tilde{\phi}) \\
a_{-}(1+a) \tilde{a}=1 & \phi_{-} \star(\epsilon+\phi \circ J) \star \tilde{\phi}=\phi_{-} \star \phi \star \tilde{\phi}=\epsilon \\
a_{+}:=\tilde{a}^{-1}=a_{-}(1+a) & \phi_{+}:=\phi_{-} \star \phi=\tilde{\phi}^{-1} \\
a_{+} \stackrel{(i)}{=} 1-\tilde{R}\left(a_{+}\left(\frac{-a}{1+a}\right)\right) & \phi_{+}=\epsilon-\tilde{\mathcal{R}}\left(\phi_{+} \star\left(\phi^{-1} \circ J\right)\right) \\
-a_{+}\left(\frac{-a}{1+a}\right) \stackrel{(i i)}{=} a_{-} a & -\phi_{+} \star\left(\phi^{-1} \circ J\right)=\phi_{-} \star(\phi \circ J)
\end{array}
$$

Proof. (i)

$$
\begin{aligned}
1-\tilde{R}\left(a_{+}\left(\frac{-a}{1+a}\right)\right) & =1-\tilde{R}\left(a_{-}(1+a)\left(\frac{-a}{1+a}\right)\right) \\
& =1+\tilde{R}\left(a_{-} a\right)=1-R\left(a_{-} a\right)+a_{-} a=a_{+}
\end{aligned}
$$

(ii)

$$
\begin{aligned}
-a_{+} \frac{-a}{1+a} & =-a_{-}(1+a) \frac{-a}{1+a} \\
& =a_{-} a
\end{aligned}
$$

The diagram (21) specializes to the following diagram in the case of renormalization: Let $g$ be the complete filtered Lie algebra of derivations in $L\left(\mathcal{H}_{F G}, \mathcal{A}\right), G$ the Lie group of characters. For $\Gamma \in \mathcal{H}_{F G}$, let $b[\phi](\Gamma)=-\left(\phi(\Gamma)+\sum_{(\Gamma)}^{\prime} \phi_{-}\left(\Gamma^{\prime}\right) \phi\left(\Gamma^{\prime \prime}\right)\right)$ be the Bogoliubov map. We have the following diagram when restricted to ker $\epsilon$

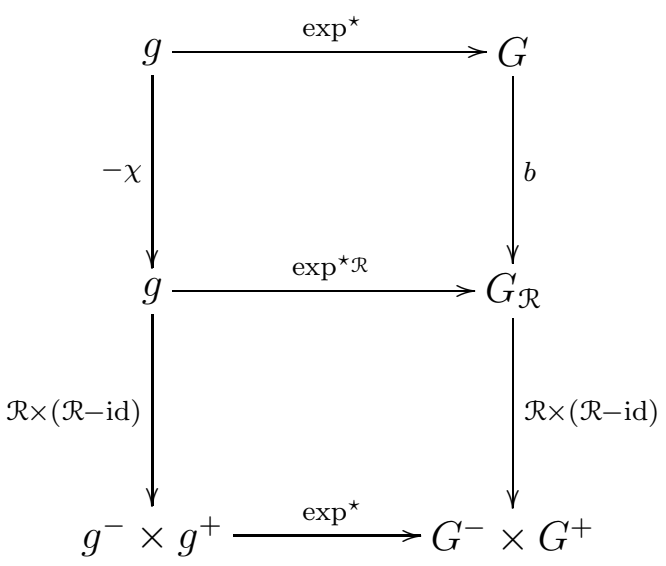

This is the reason that the map $\beta$ in (21) is called the algebraic Bogoliubov map. 


\section{$5 \quad$ Summary and Outlook}

In this work we derived, in the realm of complete filtered Rota-Baxter algebras, by simple algebraic terms a non-commutative version of Spitzer's identity. The latter is a wellknown object in the theory of random variables. The simplicity of the proofs relies on a more general result obtained in previous work by solving the recursively defined formulae of the Birkhoff decomposition of regularized characters in terms of a co-called $\mathrm{BCH}$ recursion. Initially, this was done in the Connes-Kreimer Hopf algebraic approach to renormalization theory in pQFT. This approach allowed us to derive a new forest-like formula for the renormalized character.

Also, we believe that the fact that the classical Spitzer's formula is intimately related to theory of symmetric functions and generalizations of the shuffle product might allow us to extend these connections via its non-commutative version given here.

Acknowledgements: The first author warmly thanks the Ev. Studienwerk for financial support, and the Institut Henri Poincaré for hospitality.

\section{References}

[1] M. Aguiar, Prepoisson algebras, Letters in Mathematical Physics, 54, no. 4, 263, (2000).

[2] F. V. Atkinson, Some aspects of Baxter's functional equation, J. Math. Anal. Appl., 7, 1, (1963).

[3] O. Babelon, D. Bernard, M. Talon, Introduction to classical integrable systems, Cambridge Monographs on Mathematical Physics. Cambridge University Press, Cambridge, (2003).

[4] G. Baxter, An analytic problem whose solution follows from a simple algebraic identity, Pacific J. Math., 10, 731, (1960).

[5] C. Bergbauer, D. Kreimer, The Hopf algebra of rooted trees in Epstein-Glaser renormalization, Ann. Henri Poincaré, to appear, March 2004, preprint: arXiv:hep-th/0403207.

[6] P. Cartier, On the structure of free Baxter algebras, Advances in Math., 9, 253, (1972).

[7] A. Connes, D. Kreimer, Hopf algebras, Renormalization and Noncommutative Geometry, Comm. in Math. Phys., 199, 203, (1998).

[8] A. Connes, D. Kreimer, Renormalization in quantum field theory and the Riemann-Hilbert problem. I. The Hopf algebra structure of graphs and the main theorem, Comm. in Math. Phys. 210, 249, (2000).

[9] A. Connes, D. Kreimer, Renormalization in quantum field theory and the Riemann-Hilbert problem. II. The $\beta$-function, diffeomorphisms and the renormalization group, Comm. in Math. Phys. 216, 215, (2001).

[10] K. Ebrahimi-Fard, L. Guo, D. Kreimer, Integrable Renormalization I: the Ladder case, to appear in J. Math. Phys., Feb. 2004, preprint: arXiv:hep-th/0402095. 
[11] K. Ebrahimi-Fard, L. Guo, D. Kreimer, Integrable Renormalization II: the General case, Prépublications de l'IHÉS-2004, March 2004, preprint: arXiv:hep-th/0403118.

[12] K. Ebrahimi-Fard, L. Guo, On the type and type product of dendriform algebras, May 2004, preprint: http://newark.rutgers.edu/ liguo/lgpapers.html

[13] K. Ebrahimi-Fard, L. Guo, Quasi-Shuffles, Mixable Shuffles and Hopf Algebras, May 2004, preprint: http://newark.rutgers.edu/ liguo/lgpapers.html

[14] K. Ebrahimi-Fard, Loday-type algebras and the Rota-Baxter relation, Letters in Mathematical Physics, 61, no. 2, 139, (2002).

[15] H. Figueroa, J. M. Gracia-Bondia, J. C. Varilly, Elements of Noncommutative Geometry, Birkhäuser, (2001).

[16] L. Guo, W. Keigher, Baxter algebras and shuffle products, Adv. Math., 150, no. 1, 117, (2000).

[17] L. Guo, W. Keigher, On free Baxter algebras: completions and the internal construction, Adv. Math., 151, 101, (2000).

[18] L. Guo, Baxter algebras and differential algebras, in "Differential algebra and related topics", (Newark, NJ, 2000), World Sci. Publishing, River Edge, NJ, 281, (2002).

[19] J. F. C. Kingman, Spitzer's identity and its use in probability theory, J. London Math. Soc., 37, 309, (1962).

[20] D. Kreimer, On the Hopf algebra structure of perturbative quantum field theories, Adv. Theor. Math. Phys., 2, 303, (1998).

[21] D. Kreimer, Chen's iterated integral represents the operator product expansion, Adv. Theor. Math. Phys., 3, no. 3, 627, (1999).

$[22]$ D. Kreimer, Factorization in quantum field theory: An exercise in Hopf algebras and local singularities, contributed to Les Houches School of Physics: Frontiers in Number Theory, Physics and Geometry, Les Houches, France, 9-21 Mar. 2003, March 2003, preprint: arXiv:hep-th/0306020.

[23] Ph. Leroux, Ennea-algebras, Nov 2003, preprint: arXiv:math.QA/0309213.

[24] J.-L. Loday, Dialgebras, in "Dialgebras and related operads" Springer Lecture Notes in Mathematics 1763, 2001, 7-66.

[25] D. Manchon, Hopf algebras, from basics to applications to renormalization, Comptes-rendus des Rencontres mathmatiques de Glanon 2001.

[26] G.-C. Rota, Baxter algebras and combinatorial identities. I, II., Bull. Amer. Math. Soc. 75, 325, (1969); ibid. 75, 330, (1969).

[27] G.-C. Rota, D. Smith, Fluctuation theory and Baxter algebras, Istituto Nazionale di Alta Matematica, IX, 179, (1972). Reprinted in: "Gian-Carlo Rota on Combinatorics: Introductory papers and commentaries", J.P.S. Kung Ed., Contemp. Mathematicians, Birkhäuser Boston, Boston, MA, 1995. 
[28] A. G. Reyman, M. A. Semenov-Tian-Shansky, Group theoretical methods in the theory of finite dimensional integrable systems, in: Encyclopedia of mathematical science, v.16: Dynamical Systems VII, Springer, 116, (1994).

[29] G.-C. Rota, Ten mathematics problems I will never solve, Mitt. Dtsch. Math.-Ver., 2, 45, (1998).

[30] G.-C. Rota, Baxter operators, an introduction, In: "Gian-Carlo Rota on Combinatorics, Introductory papers and commentaries", J.P.S. Kung Ed., Contemp. Mathematicians, Birkhäuser Boston, Boston, MA, 1995.

[31] M. A. Semenov-Tian-Shansky, What is a classical r-matrix?, Funct. Ana. Appl., 17, no.4., $254,(1983)$.

[32] M. A. Semenov-Tian-Shansky, Integrable Systems and Factorization Problems, Lectures given at the Faro International Summer School on Factorization and Integrable Systems (Sept. 2000), Birkhäuser 2003, Sept. 2002, preprint: arXiv: nlin.SI/0209057.

[33] F. Spitzer, A combinatorial lemma and its application to probability theory, Trans. Amer. Math. Soc., 82, 323, (1956).

[34] J. G. Wendel, A brief proof of a theorem of Baxter, Math. Scand., 11, 107, (1962). 